\title{
Dead cell phagocytosis and innate immune checkpoint
}

\author{
Kyoung Wan Yoon * \\ Department of Biotechnology, Hoseo University, Asan 31499, Korea
}

\begin{abstract}
The human body loses several billions of cells daily. When cells die in vivo, the corpse of each dead cell is immediately cleared. Specifically, dead cells are efficiently recognized and cleared by multiple types of neighboring phagocytes. Early research on cell death focused more on molecular mechanisms of cell death regulation while the cellular corpses were merely considered cellular debris. However, it has come to light that various biological stimuli following cell death are important for immune regulation. Clearance of normal dead cells occurs silently in immune tolerance. Exogenous or mutated antigens of malignant or infected cells can initiate adaptive immunity, thereby inducing immunogenicity by adjuvant signals. Several pathogens and cancer cells have strategies to limit the adjuvant signals and escape immune surveillance. In this review, we present an overview of the mechanisms of dead cell clearance and its immune regulations. [BMB Reports 2017; 50(10): 496-503]
\end{abstract}

\section{INTRODUCTION}

An adult human body consists of 60 trillion cells, where each cell conducts its roles efficiently in each tissue for its existence. The maintenance of body health needs the continuous change of aged or damaged cells with newly generated cells. Aged cells after fulfilling their respective roles, as well as damaged, infected or malignant cells, undergo programmed cell death. The clearance of impaired or damaged cells via programmed cell death is thought to be indispensable for maintaining homeostasis in living organisms (1). The proper clearance of an apoptotic cell by phagocytosis is critical for embryonic development, organ generation, tissue repairing and the achievement of an appropriate immune response. Importantly, impaired clearance of dead cells has been considered the cause of the many diseases (2-4). Accordingly, research interest of dead cell clearance is growing for greater understanding of

*Corresponding author. Tel: +82-41-540-5625; Fax: +82-41-5409538; E-mail: kwyoon@hoseo.edu

https://doi.org/10.5483/BMBRep.2017.50.10.147

Received 2 August 2017

Keywords: Dead cell clearance, Eat-me signal, Find-me signal, Immunogenic cell death, Innate immune checkpoint, Phagocytosis the networks that participate in its modulation, and all the associated mechanisms and its correlation to specific diseases.

In fact, approximately 150 billion cells (out of the 60 trillion cells in the human body) die daily (1). Interestingly, even though a number of cells are dead at any given time, experimental detection of dead cells is rare in histological studies of normal human tissues (5). And even in tissues (such as the intestine, thymus, bone marrow, and lung) where turnover rates of cells are slower, unremoved dead cells are hardly detectable. These observations suggest that the homeostatic clearance of dead cells is operated with a great degree of promptness and efficiency $(4,6,7)$. Dying cells are recognized and internalized by professional phagocytes such as macrophages and dendritic cells or neighboring nonprofessional phagocytes (8-10). Intraperitoneal injection of dexamethasone into the mouse or exposure of ionizing radiation to the whole body of the mouse results in massive thymic apoptosis of the thymus tissue. Within a few minutes after thymic cell death, the dead cells are swiftly phagocytosed by resident macrophages, and nearly all dead corpses are cleared in 24 hours $(11,12)$. Ischemia-reperfusion injury induces necrosis of renal epithelial cells in kidney tissues, but the rapid scavenging of injured cells leads to kidney regeneration and repair (13). Therefore, it is believed that efficient and prompt phagocytosis of dead cells is a critical initial step for recovery of injured tissues. In early studies, clearance of dead cells by phagocytes was thought to function simply in terms of dead cell debris scavenging. However, accumulated observations indicate that phagocytosis (once engulfment of apoptotic cells occurs) can lead to different biological consequences, depending on the circumstance of the engulfment, the dead cell types phagocytosed, or the phagocytic environment (14).

Here, we review the mechanism of apoptotic cell phagocytosis and the resulting biological response following dead cell clearance such as immune tolerance, inflammation, tissue recovery, and homeostasis.

\section{PHAGOCYTOSIS OF APOPTOTIC CELLS}

Everyday several billion cells are dying in the adult human body. Once cell death has occurred in a living organism, dead cell corpses are immediately detected and cleared by phagocytes such as macrophages and dendritic cells, rather than left in the body (15). It has been revealed that the

ISSN: 1976-670X (electronic edition)

Copyright (c) 2017 by the The Korean Society for Biochemistry and Molecular Biology

() This is an open-access article distributed under the terms of the Creative Commons Attribution Non-Commercial License (http://creativecommons.org/licenses/by-nc/4.0) which permits unrestricted non-commercial use, distribution, and reproduction in any medium, provided the original work is properly cited. 
mechanisms of dead cell clearance by phagocytes depend on the mode of cell death. Yet, there has only been progress made in the classification and analysis of apoptotic cases with regard to the mechanism of dead cell clearance following cell death. The molecular mechanisms by which phagocytes recognize and internalize apoptotic cells have been studied intensively during the last twenty years (16). Previous studies have revealed that phagocytes such as macrophages and dendritic cells recognize phosphatidylserines, which are externalized and positioned on the surface of dead cells (2). Given that phosphatidylserine functions as an indicator of when apoptotic cells should be subject for removal by phagocytes, phosphatidylserines were initially labeled as "eat-me" signals (17). In normal and healthy cells, phosphatidylserines are localized on the inner side of the surface membrane; however, when cells are dying, phosphatidylserines are expressed on the surface of apoptotic cells. Annexin V is a well-known protein that recognizes and binds phosphatidylserine in a specific manner. Therefore, Annexin $\mathrm{V}$ is usually used to identify the surface localization of phosphatidylserines in dying cells by flow cytometry analysis (18). In the early sequential stages of a cell death event, apoptotic cells can positively stained for Annexin $\mathrm{V}$ and be negatively labeled with propidium iodide. Propidium iodide used to detect DNA content by integrating into the DNA reveals that in early phase apoptotic cells, phosphatidylserine becomes exposed on the apoptotic cell surface but does not increase cell membrane permeability. So far, the molecular mechanism that promotes this exposure of phosphatidylserine on the apoptotic cell surface remains unclear. Several recent studies have uncovered that flippases are involved in the asymmetrical distribution of phosphatidylserine on the surface membranes of healthy cells. In a normal healthy cell, flippase enzymes function by maintaining phosphatidylserine localization on the inner side of surface membrane by moving phosphatidylserine from the outer leaflet to the inner leaflet of the cellular plasma membrane. Correspondingly, it has been reported that CDC50A and ATP11c play a role in the localization of phosphatidylserine on the cytoplasmic side of plasma membrane (19). It has been found that caspases cleave the flippase ATP11c upon cell death. This makes ATP11c an inactive enzyme. In addition to the inhibition of flippase, it has also been found that when cells undergo programmed cell death, caspases also activate Xkr8 and Xkr8, which play a crucial role in the effective transportation of phosphatidylserine from the inner leaflet to the outer leaflet of the plasma membrane (20). It is now thought that the inactivation and activation of ATP11c and Xkr8 enzymes collaboratively trigger the exposure of phosphatidylserine on the outer leaflet of the surface plasma membrane. The exposed phosphatidylserine serves as a physical interacting recognition marker for macrophages. Phosphatidylserine was initially identified as the unique "eat-me" signals exposed on dying cells. Subsequently, a number of proteins have been found as phosphatidylserine-binding receptors, which are expressed in phagocytes, including macrophages and dendritic cells. Several of these proteins such as Milk fat globule-EGF factor 8 (MFG-E8), MerTK, T-cell immunoglobulin mucin-3 (Tim-3) and T-cell immunoglobulin mucin-4 (Tim-4) are found to be critical for dead cell removal in vivo (21-23). Even though distinguishing roles of these proteins have yet to be elucidated in detail, it is understood that these distinct proteins mediate dead cell scavenging. Macrophages in the abdominal cavity phagocytoses dead cells, mainly via Tim- 4 and MerTK. Tim-4 induces dead cell phagocytosis via direct binding of the phosphatidylseine - an "eat-me" signal (24). MerTK mediates dead cell phagocytosis by binding to several mediating molecules such as growth arrest-specific gene 6 (Gas6) and protein $\mathrm{S}$, both of which show specific interacting activities with phosphatidylserine on the surface of dying cells. These two MerTK and Tim- 4 proteins together play essential roles in the efficient phagocytosis of dying cells by macrophages resident in the peritoneal region. In contrast, inflammatory macrophages, which are generated by the intraperitoneal administration of thioglycollate, phagocytose dead cells that are dependent on the MFG-E8 protein (21). In addition, splenic dendritic cells express Tim-3 molecules and use Tim-3 as their "eat-me" signal receptor to recognize phosphatidylserineexposed apoptotic cells. The anti-Tim-3 antibody, which functionally blocks surface Tim-3 protein, blocks the engulfment of dead cells by $\mathrm{CD}^{+}$DCs. The inhibition of phagocytosis of dead cells subsequently results in reduced cross-presentation of dead cell-associated antigens (25). Genetic mouse models targeting each molecule have exhibited distinct phenotypes, depending on the patterns of these proteins expressed on the phagocytic cells. MFG-E8 is mainly expressed in the germinal centers of the lymph nodes and spleen. Tangible body macrophages highly express MFG-E8 protein and phagocytose apoptotic lymphocytes. MFG-E8 knockout mice demonstrated inefficient phagocytic ability by macrophages in vivo. Of note, MerTK knockout mice exhibited an impaired clearance of thymic apoptotic cells (11).

Conversely, it has been reported that proteins present on the surface of healthy living cells block the engulfment by phagocytes and render the living cells unable to evade phagocytic removal. These are called as the "don't-eat-me" signals. CD47 protein is well-known "don't-eat-me" signal which has been reported to show phagocytosis-blocking activity (26). The anti-CD47 antibodies neutralize surface CD47 and has been shown to trigger phagocytosis of even living cells both in vitro and in vivo (27). Based on the phagocytosistriggering activity of anti-CD47 antibodies, anti-CD47 antibodies were found to be effective for directing cancer cells to macrophages for phagocytosis (28). This anti-CD47 antibodies are under development as an immune modulatory anti-cancer drug.

For cells that have gone through apoptosis and require prompt phagocytosis and removal by macrophages, it is indispensable that phagocytes migrate to these dead cells. It 
has been found that dying cells secret a number of molecules such as chemo-attractants in order to recruit phagocytes to the site. The releasing factors from apoptotic cells are referred to as "find-me" signals. In several studies, it has been reported that ATP and lysophosphatidylcholine secreted from apoptotic cells play a critical role in recruiting phagocytes, including macrophages (29). However, the molecular mechanisms of these "find-me" signaling molecules to enable recruitment of phagocytes in vivo remain unclear.

\section{DEAD CELL SCAVENGING IN VIVO}

Given that dead cell clearance by macrophage occurs swiftly and efficiently in living organisms, few dead cells are observed uncleared in any tissue. Although a significant number of apoptotic cells stained with TUNEL can be detected in the thymus, apoptotic cells are mostly observed inside of resident macrophages (5). Based on this biological phenomenon, one question has been raised: why are dead cells removed almost immediately after cell death without lingering as dead cell corpses? Using genetic mouse models that are deficient in genes involved in the phagocytosis of dead cells, many studies have revealed the significance of apoptotic cell clearance in living organisms. For example, in MFG-E8 and MerTK knockout mice, the in vivo phagocytosis of apoptotic cells are visibly impaired. Consequently, uncleared dead cell corpses induce symptoms that are the hallmark of autoimmune disease, including significant elevations of serum anti-DNA antibody and anti-nuclear antibody titers (3). From these findings, dead cell clearance by phagocytes is thought to play a critical role in the maintenance of immune tolerance to self-antigen. This biological phenomenon, of promptly clearing away of dead cells, is recognized as a means of preventing autoantigens from being released by dead cells in order to block the abnormal activation of autoimmune response. Still, several others have offered another interpretation; they posit that phagocytes might play an active role in inducing immune tolerance to self-antigens through the phagocytosis of apoptotic cells. Phagocytes that engulf apoptotic cells are thought to actively transduce negative or immune tolerating signals to self-reactive $\mathrm{T}$ cells by presenting engulfed autoantigens, which have been loaded on MHC. Multicellular organisms exhibit continuous cellular turnover of dead cells daily and continuously. Apoptotic cells residing in specific tissues contain tissue-specific self-antigens. Therefore, the uncleared dead cell corpses could provide sources of self-antigens for antigen-presenting cells such as macrophages and dendritic cells. When apoptosis is stimulated by the administration of dead cells, either intravenously or intraperitoneally, T-cell responses to self-antigens generated from apoptotic cells become diminished (30). Subsequently splenic dendritic cells engulf the administered apoptotic cells and self-antigens from the apoptotic cells are presented to resident $T$ cells for the activation of immune tolerance. Apoptotic cells may themselves induce immune tolerance. Although splenic dendritic cells are stimulated by activation signals such as costimulatory signals, the cross-presentation of self-antigens might result in the removal of self-antigen-specific T cells (31). Interestingly, under specific physiological conditions, phagocytosis of apoptotic cells could lead to the cross-presentation of self-antigens by phagocytes, resulting in the activation of T-cell responsiveness to self-antigens. $T$ cell activation against apoptotic cellassociated antigens has been broadly studied in the field of immuno-oncology. It has been reported that apoptotic cancer cells can trigger cancer cell antigen-specific T-cell activationwhether the induction of apoptosis of tumor cells occurs in vivo or whether induction is via direct injection of apoptotic cells (32). Accordingly, the efficiency of tumor vaccination using apoptotic cancer cells mostly depends on the type of cellular apoptosis induced in cancer cells. It has been reported that the expression of the calreticulin protein on the apoptotic cancer cell efficiently leads to anti-tumor immunity (33), suggesting that calreticulin may modulate phagocytosed tumor antigen-induced immune activation. In other studies, it has been observed that the injection of stable cells artificially expressing CD1d, which can load $\alpha$-galactosylceramide and other tumor antigens, can boost tumor immunity (34). In these studies, CD1d expressing cells were believed to undergo apoptosis in vivo and hagocytosed by dendritic cells. The engulfed dendritic cells express tumor antigens by crosspresentation, which activate tumor antigen-specific cytotoxic $T$ lymphocytes. This activation of cytotoxic $\mathrm{T}$ cells by tumor antigen-presenting dendritic cells occurs cooperatively with the activation of natural killer $\mathrm{T}$ cells. Not surprisingly, the detailed molecular mechanisms of immune response to apoptotic cell- or dead tumor cell-associated antigens need to be elucidated. Such studies can provide new insights for controlling phagocytosed apoptotic cell-induced immune responses as well as inform future cancer immune therapeutic strategies.

\section{TYPES OF PHAGOCYTES FOR DEAD CELL ENGULFMENT}

In living organisms, professional phagocytes such as dendritic cells and macrophages mainly act by engulfing and controlling the subsequent immune response. In particular, different types of phagocytes have been studied in an attempt to uncover the actual players, and classify those players, that contribute to immune modulation. Each tissue possesses tissue-specific phagocytes, including macrophages and dendritic cells; and these tissue-resident phagocytes internalize apoptotic cells. Recent studies have found that there are distinct functions in immune modulation in which phagocytes participate; and their corresponding functions depend, in part, to the given tissue in which they reside as well as to the different subpopulations of phagocytes that exist even within same tissue. In tissues, phagocytes have been found to be heterogeneous, 
demonstrating functional diversity (35). In other words, specific subpopulations of phagocytes are primarily responsible for dead cell clearance in tissues. In fact, several subpopulations that reside in lymph nodes and the spleen have been found to play a critical role in the phagocytosis of dying cells, in addition to the modulation of immune tolerance. As discussed earlier, intravenously administrated apoptotic cells do not activate an immune response to apoptotic cell-associated self-antigens, resulting in immune tolerance. For instance, specific subpopulations of macrophages and/or marginal metallophilic macrophages reside in the marginal region of spleen. Since the marginal zone of the spleen is the site blood passage, splenic marginal zone macrophages have been found to phagocytose apoptotic cells in the blood. The critical role of marginal zone macrophages in dead cell phagocytosis and in tolerance induction has been identified by work in CD169DTR mice. In CD169-DTR mice, the marginal zone of macrophages was deleted with DT injection (30). Dendritic cells expressing CD103 and CD11c are also residents in the splenic marginal zone. These dendritic cells contribute to the crosspresentation of antigens, which originate in many cells, including apoptotic cells and CD8-positive $\mathrm{T}$ cells. This suggests that cooperative immune modulation occurs between dendritic cells and macrophages (36). Apoptotic cells induce immune tolerance when cell death occurs. The underlying molecular mechanisms of immune tolerance have been described in previous reports. When apoptotic cells are intravenously injected into the mouse, metallophilic macrophages in the spleen are stimulated to express CCL22. CCL22 expression in splenic macrophages leads to the recruitment and induction of FoxP3-positive regulatory $\mathrm{T}$ cells (Treg cells). Alternatively, in cancer, a number of apoptotic cells in the tumor tissue can result in an immunological activation in response to tumor cell-related antigens; this, in turn, triggers the development of immunity towards the tumor cells. The subsets of dendritic cell and macrophage involved in anticancer immune activation have been well-characterized. When mice are subcutaneously injected with dead tumor cells, CD169-positive macrophages in the lymphatic cavity of the lymph node phagocytose apoptotic cells or dead cell debris in the lymph. The dead-cell engulfed macrophages triggers activation of an immunological response to neoantigens. CD169-positive macrophages consist of two subsets; CD11cnegative and CD11c-positive macrophages. In the border region between the nasal cavity and $\mathrm{T}$ cell zone, CD11cpositive macrophages present neoantigens originating from the dead tumor cell to CD8-positive T cells by cross-presentation. Given that immunological activation of apoptotic cell-related antigens are important for the treatment of cancer and autoimmune diseases, the characterization of macrophage subpopulations involved in dead cell antigen-responsive macrophages are essential subjects worth investigating further.

During the last few decades, cell death had been considered the final event of the cellular life cycle, and dead cell corpses had only been viewed as debris, simply to be removed by phagocytes. In more recent studies, the notion that the cell death process may actually serve as a starting point, in stimulating various subsequent biological processes instead, has come to light. Additionally, it has been revealed that dying cells actively release physiological substances which activate several biological processes. Among the more well-characterized physiological responses triggered by cell death that is inflammation. The high mobility group box protein 1 (HMGB1) is a dead cell-releasing factor, which stimulates the immune response. HMGB1 protein was initially identified as a nuclear factor. However, nuclear HMGB1 is also released when cells die via an alternative mechanism to apoptosis (37). Once HMGB1 is released, HMGB1 proteins causes an inflammatory response by targeting macrophages and dendritic cells (38). C-Type Lectin Domain Family 4 Member E (CLEC4E) Mincle, a C-type lectin, is expressed on the surface of macrophages. CLEC4E receptor recognizes the soluble factor, SAP130, released from dying cells to initiate inflammation (39). The inflammation-inducing factors, which originate from dying cells, are referred to as damage-associated molecular patterns (DAMPs). Furthermore, in many lines of studies, it has been uncovered that substances derived from dying cells contribute to tissue repair and organ regeneration. When liver cells get damaged such as via oxidative stress, dying hepatic cells have been known to release IL-11. IL-11 stimulates the neighboring normal cell to influence the regeneration of liver tissue. Several studies have reported that semaphorin 3E is highly expressed in apoptotic hepatic cells and modulates liver fibrogenesis, repair and regeneration (40). Still, other studies have demonstrated that chronic liver damage induces dead cell phagocytosis and upregulation of Wnt3a in macrophages, resulting in liver regeneration. Even though an association between cell death and the regeneration of injured tissue has been established, the specific molecular mechanisms have not been elucidated. Therefore, further research detailing the mechanisms of dead cell-induced tissue regeneration is anticipated.

\section{APOPTOTIC AND NON-APOPTOTIC CELL DEATH}

Cell death has been intensively studied in the last few decades. Programmed cell death (called apoptosis) was considered to be the main type of cell death thought to occur in vivo. Apoptosis is accompanied by the activation of the caspase cascade signaling mechanism, with the eventual degradation of intracellular substrates. Therefore, apoptosis is considered an active form of cell death, which is regulated by active molecules. Given that caspases are activated in apoptotic cells, detecting active caspases has been widely used to analyze apoptotic cells. Additionally, the terminal deoxynucleotidyl transferase (TdT) dUTP Nick-End Labeling (TUNEL) assay has also been well-established for detecting apoptotic cells in vitro and in situ (41). By using these assays, 
programmed cell death could be detected in multiple organs during embryonic development as well as during normal tissue turnover. A high technique to detect apoptotic cells in vivo in the real time has also been developed. Based on this newer assay, the dynamic turnover of apoptotic cells in vivo and its effects on the neighboring cells have been extensively characterized, especially during embryogenesis (42). Nonapoptotic cell death (called necrosis) is a type of cell death, which is caused by other physical stimuli as well as heat. And pathological cell death caused by diseases is also considered a passive mode of cell death. Necrotic cell death exhibits similar morphological characteristics of dying cells. Given that necrotic cell death is a passive form of cell death, it was assumed that no particular molecular mechanism played a part in necrotic cell death. Yet, recent studies have found that necrotic cell death has several subtypes, which are actively regulated by specific molecules. Receptor interacting serine/ threonine kinase 1 (RIPK1)/receptor interacting serine/threonine kinase 3 (RIPK3) and mixed lineage kinase domain-like (MLKL) have been identified to play an active role in some forms of necrosis, a form of cell death. These subtypes of necrosis are referred to necroptosis (43). Another new type of necrotic cell death, caspase-1-mediated cell death has been identified, now referred to as pyrotosis (44). These novel subtypes of nonapoptotic cell deaths exhibit the typical necrotic morphological features. However, necroptosis or pyroptosis were found to contribute to different pathological states of various diseases. More recently, an additional type of cell death (which requires iron ions), referred to as ferroptosis, has been identified (45). The molecular mechanisms of this newly identified cell death has been studied in vitro. Although the in vivo role of the associated molecules has also been studied using genetically modified mouse models, their physiological functions in relation to this new form of cell death are still gradually being elucidated. As there is no satisfactory method of detection for new types of cell death, in vivo methodologies for studying new cell deaths is challenging. It is believed that the mechanisms of dead cell removal by phagocytes depends on the specific type of cell death that takes place. Moreover, dendritic cells and macrophages determine the appropriate response to dead cells, depending on the specific mode of cell death. In order to follow the physiological and pathological consequences of a dying cell in vivo, it will be necessary to investigate how dendritic cells and macrophages respond to dead cells according to the different modes of cell death.

\section{INNATE IMMUNE CHECKPOINT FOR CANCER THERAPY}

Recently, T cell immune checkpoint inhibitors for cancer therapy have achieved great outcomes toward reaching the goal of treating cancer successfully. However, the effect of T cell immune checkpoint inhibitors is very limited, in only a specific population of patients. Patients that do not respond to these drugs show non-T cell-inflamed tumors. And the removal of cancer cells by $T$ cells is significantly dependent on the activation of innate immunity. Therefore, additional targeting of innate immunity may provide a strategy for developing improved therapies against cancers. The TAM receptor tyrosine kinases, including TYRO3 protein tyrosine kinase (TYRO3), AXL receptor tyrosine kinase (AXL), and MER receptor tyrosine kinase (MERTK) have been studied as innate immune checkpoint proteins involved in critical steps of anti-tumor immunity (46). Efferocytosis refers to the process by which dead cells are cleared by phagocytes. TAM receptor tyrosine kinases have been known to inhibit the activity of dendritic cells, which is one of the major phagocytes that dysregulates the production of chemokines, eventually resulting in the immune evasion of tumor cells. Therefore, employing a TAM signaling blockade strategy for anti-cancer immune therapy is one of great focus, as it may potentially enhance the cooperative effects between anti-tumor adaptive immunity and immune checkpoint blockade targeting $\mathrm{T}$ cells. Current immune checkpoint inhibitors such as anti-PD-1, anti-CTLA-4, or anti-PD-L1 antibodies show excellent promising outcomes in producing an anti-tumor effect in melanoma and lung cancer patients (47). Moreover, modulating the strategy of immune cells has led the long-lasting anti-tumor immunity. However, a limited population of patients show responsiveness to $T$ cell immune checkpoint therapy. Studies to understand the distinct mechanisms between responsive and unresponsive patients continue to intensify. In order to overcome the narrow response to $\mathrm{T}$ cell immune checkpoint therapy, co-treatments with multiple reagents have been applied. For example, combinational therapy with rituximab and anti-PD1 antibody for follicular lymphoma has shown a $66 \%$ response rate. Although this therapeutic outcome is remarkable for some patients, the use of immunotherapies is still limited to a small portion of patients. Unresponsiveness to $\mathrm{T}$ cell immune checkpoint inhibitors is mostly caused by a loss of $\mathrm{T}$ cell response in the tumor tissue. Therefore, it is important to target other novel immune inhibitory pathways that can circumvent the failure of the T cell immune checkpoint therapy. Several pathways have been considered as target candidates. Importantly, such alternative therapies (representing new target candidates) should not result in collateral damage to the patient. Blockade of receptor tyrosine kinases (RTKs) can be achieved with small molecule inhibitors, ligand blockers, and other inhibitory strategies. Recently, a new cancer therapeutic strategy targeting the TAM tyrosine kinase receptors has garnered attention, and a number of small molecules inhibiting TAM receptors are in development (46). A small molecule inhibitor of $\mathrm{AXL}, \mathrm{BGB} 324$ has shown significant regression of the growth of multiple cancer cell lines. BGB324 also inhibited migration, metastasis, and angiogenesis in a breast cancer xenograft mouse model. Based on the preclinical effects, a Phase 1 trial has subsequently been completed. In the mouse tumor model system, it was found to activate AXL 
tyrosine kinase, which leads to resistance of erlotinib, a tyrosine kinase inhibitor (TKI) targeting epidermal growth factor receptor (EGFR). Drug sensitivity to erlotinib TKI increased upon genetic deficiency or small molecule inhibition of AXL. EGFR-mutant lung cancer samples obtained from patients with acquired resistance to TKIs showed increased expression of the AXL protein level. Several MerTK-specific small molecule inhibitors are under development. In mouse models of non-small-cell lung cancer (NSCLC), UNC2025, the MerTK small molecule inhibitor demonstrated significant suppression of tumor growth. UNC2025 sufficiently induced the death of NSCLC tumor cells carrying oncogenic mutations such as K-Ras and EGFR mutations (48). Another MerTK inhibiting small molecule, UNC1666, is also in development. MerTK inhibitors indirectly generates an anti-tumor effect by targeting other proteins such as fms-related tyrosine kinase 3 (FLT3). Ligand trapping and monoclonal antibodies provide yet another alternative method of treatment. A GAS6 high affinity engineered extracellular domain of the AXL tyrosine kinase receptor can inhibit the ligand from functioning by sequestering AXL. In a similar manner, the soluble ligand-binding protein fragment of MerTK can disrupt GAS6 function, leading to its impairment, apoptosis and eventual engulfment by macrophages. Monoclonal antibodies targeting TAM receptors have recently been reported. Treatment of cancer cells with TAM receptor tyrosine kinase can inhibit survival and transformation of cancer cells. Their actions on MerTK to engender an effect on the innate immune system to bring about immune activation. Therefore, TAM receptor tyrosine kinases represent good targets for pharmacologic treatment. An anti-tumorigenic function of the TAM signaling pathway has been found in colon carcinoma model induced by inflammation. Several studies have observed that TAM inhibition can yield different outcomes depending on the severity of inflammation, the amount of TAM RTK-expressing cells, and the phase of tumor progression. Studies using TAM tyrosine kinases inhibitors ought to reveal more detailed mechanisms by which TAM signaling contributes to tumor killing. In order to evaluate TAM inhibitors as an anti-tumor therapeutic drug, all the essential factors of systemic blocking of TAM inhibitors should be considered: the effects arising from lengthy duration of treatment, the degenerative degree of photoreceptor and vision that can result, and extratumoral homeostasis. Thus, new cancer immunotherapeutic strategies, targeting innate immune checkpoints, might trigger the recruitment and activation of immune cells in patients.

\section{IMMUNOGENEIC CELL DEATH}

Immunogenic cell death is a prevalent theme in tumor biology. Detection of the ajuvanticity of immunogenic dead cells occurs via the unique damage-associated molecular patterns. This simplified characterization of molecular phenomenon is based on immune surveillance against the primordial system. As a result, the concept of immunogenic cell death cannot be applied to anticancer immune surveillance. In the context of microorganism infection, studies report that viruses and bacteria can develop various strategies to evade immune surveillance by masking the recognition of damage-associated molecular patterns. The molecules, which multiple microorganisms use for immune evasion include orthologues of antiapoptotic proteins such as the BCL-2 family, negative regulating proteins of autophagy, dephosphorylating proteins of elF2A, inflammasome-inhibiting proteins, and/or blocking factors of type I IFN signaling. Once the host is infected with pathogens, the host determines the adjuvanticity of infected cell death. Adjuvanticity rather than antigenicity controls immunogenicity. Malignant transformation of cells also leads selective adjuvaticity in patients and high adjuvaticity can assist with cancer immunosurveillance. Once neoplastic cells escape immune detection and removal, malignant cells can develop in the tumor. This might be due to inhibition of damage-associated molecular patterns, resulting in the subsequent immunological responses. Even though advanced cancers show high antigenicity, malignant tumor cells exhibit various mechanisms to lower immunogenicity. Based on their escape mechanisms, cancer cells impair the efficiency of multiple anti-cancer therapies, including chemotherapy, radiotherapy and immunotherapy. Therefore, restoration of the immunogenicity of cancer cells might reconstitute their adjuvanticity, eventually achieving therapeutic success (49).

\section{CONCLUSIONS}

Here, we provide an overview of the molecular mechanisms of apoptotic cell phagocytosis and its pathophysiological significance. So far, the mechanisms of cell death, classification of subtype of cell death, disease relevance of cell death have been intensively studied. Based on recent accumulated observations, indicating the significance of 'post-apoptotic biological events', the interest of studying dead cell clearance and the subsequent biological consequences continues to grow. However, to understand the physiological role of dead cell clearance, we need to overcome a number of challenges such as: the identification of various cell death types, the development of analytical methods for determining their biological significance, and the development of analytical techniques for determining the mechanisms that modulate a physiological response after cell death and dead cell clearance. As the subsequent biological events after dead cell clearance depends on the cell death modes, the types of engulfing phagocytes, tissue specificity, and phagocytic microenvironment, precise understanding of dead cell clearance will be important. A detailed verification of the biological role of dead cell clearance will provide a deeper knowledge to enable our understanding of the causes and consequences of impairment of dead cell clearance in multiple human diseases and conditions. 


\section{CONFLICTS OF INTEREST}

The authors have no conflicting interests.

\section{REFERENCES}

1. Bianconi E, Piovesan A, Facchin F et al (2013) An estimation of the number of cells in the human body. Ann Hum Biol 40, 463-471

2. Nagata S, Hanayama R and Kawane K (2010) Autoimmunity and the clearance of dead cells. Cell 140, 619-630

3. Green DR, Oguin TH and Martinez J (2016) The clearance of dying cells: table for two. Cell Death Differ 23, 915-926

4. Elliott MR and Ravichandran KS (2010) Clearance of apoptotic cells: implications in health and disease. J Cell Biol 189, 1059-1070

5. Mcllroy D, Tanaka M, Sakahira H et al (2000) An auxiliary mode of apoptotic DNA fragmentation provided by phagocytes. Genes Dev 14, 549-558

6. Lee CS, Penberthy KK, Wheeler KM et al (2016) Boosting Apoptotic Cell Clearance by Colonic Epithelial Cells Attenuates Inflammation In Vivo. Immunity 44, 807-820

7. Sunaga $H$, Matsui $H$, Ueno M et al (2013) Deranged fatty acid composition causes pulmonary fibrosis in Elovl6deficient mice. Nat Commun 4, 2563

8. Poon IK, Lucas CD, Rossi AG et al (2014) Apoptotic cell clearance: basic biology and therapeutic potential. Nat Rev Immunol 14, 166-180

9. Ravichandran KS and Lorenz U (2007) Engulfment of apoptotic cells: signals for a good meal. Nat Rev Immunol 7, 964-974

10. Lauber K, Bohn E, Krober SM et al (2003) Apoptotic cells induce migration of phagocytes via caspase-3-mediated release of a lipid attraction signal. Cell 113, 717-730

11. Scott RS, McMahon EJ, Pop SM et al (2001) Phagocytosis and clearance of apoptotic cells is mediated by MER. Nature 411, 207-211

12. Yoon KW, Byun S, Kwon E et al (2015) Control of signaling-mediated clearance of apoptotic cells by the tumor suppressor p53. Science 349, 1261669

13. Bonventre JV and Yang L (2011) Cellular pathophysiology of ischemic acute kidney injury. J Clin Invest 121, 4210-4221

14. Arandjelovic S and Ravichandran KS (2015) Phagocytosis of apoptotic cells in homeostasis. Nat Immunol 16, 907-917

15. Fond AM and Ravichandran KS (2016) Clearance of Dying Cells by Phagocytes: Mechanisms and Implications for Disease Pathogenesis. Adv Exp Med Biol 930, 25-49

16. Elliott MR and Ravichandran KS (2016) The Dynamics of Apoptotic Cell Clearance. Dev Cell 38, 147-160

17. Segawa K and Nagata S (2015) An Apoptotic 'Eat Me' Signal: Phosphatidylserine Exposure. Trends Cell Biol 25, 639-650

18. van Engeland M, Nieland LJ, Ramaekers FC et al (1998) Annexin V-affinity assay: a review on an apoptosis detection system based on phosphatidylserine exposure. Cytometry 31, 1-9
19. Segawa K, Kurata S, Yanagihashi Y et al (2014) Caspasemediated cleavage of phospholipid flippase for apoptotic phosphatidylserine exposure. Science 344, 1164-1168

20. Suzuki J, Denning DP, Imanishi E et al (2013) Xk-related protein 8 and CED-8 promote phosphatidylserine exposure in apoptotic cells. Science 341, 403-406

21. Hanayama R, Tanaka M, Miwa K et al (2002) Identification of a factor that links apoptotic cells to phagocytes. Nature 417, 182-187

22. Rothlin CV, Carrera-Silva EA, Bosurgi L et al (2015) TAM receptor signaling in immune homeostasis. Annu Rev Immunol 33, 355-391

23. Freeman GJ, Casasnovas JM, Umetsu DT et al (2010) TIM genes: a family of cell surface phosphatidylserine receptors that regulate innate and adaptive immunity. Immunol Rev 235, 172-189

24. Miyanishi M, Tada K, Koike M et al (2007) Identification of Tim4 as a phosphatidylserine receptor. Nature 450, 435-439

25. Nakayama M, Akiba H, Takeda K et al (2009) Tim-3 mediates phagocytosis of apoptotic cells and crosspresentation. Blood 113, 3821-3830

26. Kinchen JM and Ravichandran KS (2008) Phagocytic signaling: you can touch, but you can't eat. Curr Biol 18, R521-524

27. Chao MP, Alizadeh AA, Tang C et al (2010) Anti-CD47 antibody synergizes with rituximab to promote phagocytosis and eradicate non-Hodgkin lymphoma. Cell 142, 699-713

28. Edris B, Weiskopf K, Volkmer AK et al (2012) Antibody therapy targeting the CD47 protein is effective in a model of aggressive metastatic leiomyosarcoma. Proc Natl Acad Sci U S A 109, 6656-6661

29. Medina CB and Ravichandran KS (2016) Do not let death do us part: 'find-me' signals in communication between dying cells and the phagocytes. Cell Death Differ 23, 979-989

30. Miyake Y, Asano K, Kaise H et al (2007) Critical role of macrophages in the marginal zone in the suppression of immune responses to apoptotic cell-associated antigens. J Clin Invest 117, 2268-2278

31. Liu K, Iyoda T, Saternus $M$ et al (2002) Immune tolerance after delivery of dying cells to dendritic cells in situ. J Exp Med 196, 1091-1097

32. Asano K, Nabeyama A, Miyake $Y$ et al (2011) CD169positive macrophages dominate antitumor immunity by crosspresenting dead cell-associated antigens. Immunity 34, 85-95

33. Chao MP, Jaiswal S, Weissman-Tsukamoto R et al (2010) Calreticulin is the dominant pro-phagocytic signal on multiple human cancers and is counterbalanced by CD47. Sci Transl Med 2, 63ra94

34. Fujii S, Goto A and Shimizu K (2009) Antigen mRNAtransfected, allogeneic fibroblasts loaded with NKT-cell ligand confer antitumor immunity. Blood 113, 4262-4272

35. Gordon S, Pluddemann A and Martinez Estrada F (2014) Macrophage heterogeneity in tissues: phenotypic diversity and functions. Immunol Rev 262, 36-55

36. Qiu CH, Miyake $Y$, Kaise $H$ et al (2009) Novel subset of CD8\{alpha\} + dendritic cells localized in the marginal 
zone is responsible for tolerance to cell-associated antigens. J Immunol 182, 4127-4136

37. Rovere-Querini P, Capobianco A, Scaffidi P et al (2004) HMGB1 is an endogenous immune adjuvant released by necrotic cells. EMBO Rep 5, 825-830

38. Dumitriu IE, Baruah P, Valentinis B et al (2005) Release of high mobility group box 1 by dendritic cells controls $T$ cell activation via the receptor for advanced glycation end products. J Immunol 174, 7506-7515

39. Yamasaki S, Ishikawa E, Sakuma M et al (2008) Mincle is an ITAM-coupled activating receptor that senses damaged cells. Nat Immunol 9, 1179-1188

40. Yagai T, Miyajima A and Tanaka M (2014) Semaphorin 3E secreted by damaged hepatocytes regulates the sinusoidal regeneration and liver fibrosis during liver regeneration. Am J Pathol 184, 2250-2259

41. Gavrieli Y, Sherman Y and Ben-Sasson SA (1992) Identification of programmed cell death in situ via specific labeling of nuclear DNA fragmentation. J Cell Biol 119, 493-501

42. Nonomura K, Yamaguchi $Y$, Hamachi M et al (2013) Local apoptosis modulates early mammalian brain development through the elimination of morphogen-producing cells. Dev Cell 27, 621-634

43. Pasparakis $M$ and Vandenabeele $P$ (2015) Necroptosis and its role in inflammation. Nature 517, 311-320

44. Lamkanfi M and Dixit VM (2014) Mechanisms and functions of inflammasomes. Cell 157, 1013-1022

45. Yang WS, SriRamaratnam R, Welsch ME et al (2014) Regulation of ferroptotic cancer cell death by GPX4. Cell 156, 317-331

46. Vouri M and Hafizi S (2017) TAM Receptor Tyrosine Kinases in Cancer Drug Resistance. Cancer Res 77, 2775-2778

47. Byun DJ, Wolchok JD, Rosenberg LM et al (2017) Cancer immunotherapy - immune checkpoint blockade and associated endocrinopathies. Nat Rev Endocrinol 13, 195-207

48. DeRyckere D, Lee-Sherick AB, Huey MG et al (2017) UNC2025, a MERTK Small-Molecule Inhibitor, Is Therapeutically Effective Alone and in Combination with Methotrexate in Leukemia Models. Clin Cancer Res 23, 1481-1492

49. Galluzzi L, Buque A, Kepp O et al (2017) Immunogenic cell death in cancer and infectious disease. Nat Rev Immunol 17, 97-111 